\title{
MIMO Fuzzy Control for Autonomous Mobile Robot
}

\author{
Thoa T. Mac ${ }^{1,2}$, Cosmin Copot $^{1}$, Robin De Keyser ${ }^{1}$, Trung D. Tran ${ }^{2}$, and Thich Vu ${ }^{2}$ \\ ${ }^{1}$ Department of Electrical Energy, Systems and Automation, Ghent University \\ ${ }^{2}$ School of Mechanical Engineering, Hanoi University of Science and Technology (HUST)
}

\begin{abstract}
This paper addresses the design and implementation of multi-input multi-output (MIMO) fuzzy control for mobile robot. Firstly, MIMO fuzzy control is apply to track different desired trajectories. Secondly, the controller performs on robot for navigation purpose to avoid obstacles and reach defined target. The proposed MIMO fuzzy controller was investigated based on several conducted MATLAB simulation scenarios for mobile robot. The simulation results are presented to demonstrate the effectiveness of our new control algorithm.
\end{abstract}

Index Terms - trajectory tracking, path planning, mobile robot navigation, MIMO fuzzy control

\section{INTRODUCTION}

An Autonomous Mobile Robot is an artificial intelligent vehicle that is capable of traveling in different situation. In which, following defined trajectory and navigation itself to avoid obstacles and move to destination point is key issue. It is observed that the human beings do not need precise, numerical information input to make a decision, but they are able to perform highly adaptive control. Human has a remarkable capability to perform a wide variety of physical and mental tasks without any explicit measurements or computations. Examples of everyday tasks are parking a car, driving in different terrains, avoiding static and dynamics obstacles. For human, the ability to navigate is eminent however, for robot; this kind of task is complex and challenge. To recognize the trajectories; obstacles and targets, robot must consider the information from the working environment obtained from its sensor or vision system. Various methods have been applied to solve motion control problems. They are classified into model based methods and artificial methods those employ fuzzy logic, neural networks, genetic algorithms or hybrid of these approaches.

Among all the suggested methods for reactive navigation, fuzzy logic controller has been found to be the most attractive. The theory of fuzzy logic systems is inspired by the remarkable human capability to operate on and reason with perception-based information. The rule-based fuzzy logic provides a scientific formalism for reasoning and decision making with uncertain and imprecise information. It can be implemented in

Manuscript received December 21, 2014; revised March 11, 2015. hardware, software, or a combination of both. Fuzzy logic approach to control problems mimics how a person would make decisions. The main advantages of a fuzzy navigation strategy lie in the ability to extract heuristic rules from human experience, and to obviate the need for an analytical model of the process. It is tolerant to noise and error in the information coming from the sensory system, and most importantly it is a factual reflection of the behavior of human expertise. This kind of controller is a form of artificial decision-making system. Several tasks of robot are investigated by using fuzzy logic control. Tracking trajectory is performed on mobile robot in [1] [2] [9]. In the first paper, the authors propose a path following approach based on a fuzzy-logic set of rules, which emulates the human driving behavior. The input to the fuzzy system is represented by approximate information concerning the next bend ahead the vehicle; the corresponding output is the cruise velocity that the vehicle needs to attain in order to safely drive on the path. [2] It has different approach with a heuristic fuzzy logic controller has been designed based on a model-free approach. Good tracking control performance was also obtained from the proposed controller. [3] proposes a reinforcement ant optimized fuzzy controller design method and applies it to wheeled-mobile-robot wallfollowing control under reinforcement learning environments. Navigation for autonomous vehicle is researched in [4]-[6]. Hierarchical fuzzy design decomposes the controller into three particular fuzzy systems: fuzzy steering, fuzzy linear velocity control and fuzzy angular velocity control, so that manual construction of each rule base becomes feasible and easy [5]. Fuzzy controller is integrated in [7] to avoid dynamics obstacle. The hallmark of [8] investigates the application of an adaptive neuro-fuzzy inference system (ANFIS) to path generation and obstacle avoidance for an autonomous mobile robot in a real world environment. [9] a control structure that makes possible the integration of a kinematic controller and an adaptive fuzzy controller for trajectory tracking is developed for nonholonomic mobile robots.

However, it is clearly that real life application of mobile robot involves a multivariable input-output 
system, it is necessary to find suitable MIMO controller [11]-[14]. A hybrid indirect and direct adaptive fuzzy output tracking control schemes are developed for a class of nonlinear (MIMO) systems. Being the auxiliary compensation, $\mathrm{H}^{\infty}$ control and sliding mode control are designed to suppress the influence of external disturbance and remove fuzzy approximation error, respectively so that the system maintain a good tracking performance. [14] designs of wall tracking robot mobile based on intelligent controller, MIMO Fuzzy Logic controller, that it's equipped with three ultrasonic sensors. The simulation is performed in MATLAB Fuzzy Logic Toolbox

In this research, design of new MIMO fuzzy logic controller for tracking trajectory and navigation behavior of mobile robot is presented. System has two fuzzy controllers that each of them has two inputs and two outputs for tracking task and four inputs and two outputs for autonomous navigation task. The information from vision system forms input to the controller while outputs controlling the speed of motors. The fuzzy controller is designed on MATLAB Simulink environment and Fuzzy Toolbox. More details could be found in following sections. The paper is organized as follows: Section II presents the model of mobile robot. The control design of the path-following strategies is depicted in section III. Next in section IV, the navigation controller for mobile robot is descripted. The simulation results for each algorithm are showed respectively in section III and IV, followed by a conclusion section where the main outcome of this work is summarized.

\section{RoBOT MODEL}

The first step to a kinematic model of the robot is to express constraints on the motions of individual wheels. To test the algorithm, a simple model of robot is chosen. The robot has a differential drive system with two independent wheels and a caster for stability. The first constraint enforces the concept of rolling contact that the wheel must roll when motion takes place in the appropriate direction. The second constraint enforces the concept of no lateral slippage, that the wheels have pure rolling and non-slipping conditions during the motion in wheel plane. The position of the mobile robot in the global frame $\mathrm{X}(\mathrm{O}) \mathrm{Y}$ can be defined by the position of mass center of robot $\mathrm{C}(\mathrm{x}, \mathrm{y})$ which is the center of mobile gear; and the angle between the local frame $\mathrm{X}_{\mathrm{m}} \mathrm{CY}_{\mathrm{m}}$ and the global frame $(\theta)$.

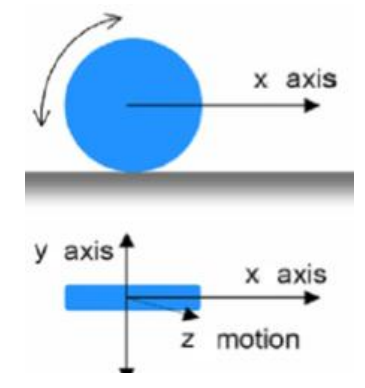

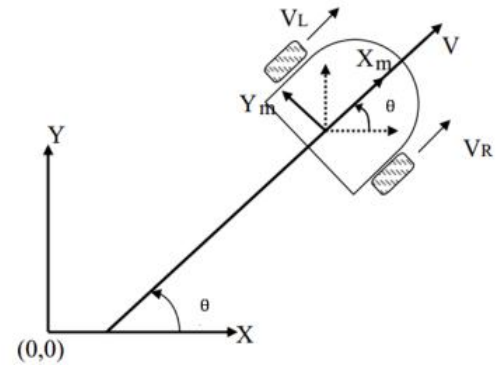

Figure 1. Ideal wheel and Kinematics model of robot.

The center of mobile robot $(x, y, \theta)$ is expressed in the inertial coordinate frame. The state of robot is defined by its position $(\mathrm{x}, \mathrm{y})$ and orientation $(\theta)$ and by the speeds of the mobile robot. A simple structure of robot is showed in Fig. 1. Supposing that the robot moves on a plane with linear and angular velocities, the state vector can be express as $\dot{q}=(\dot{x}, \dot{y}, \dot{\theta})$. Path planning under kinematic constraints is transformed into a pure geometric problem. Mobile robot motion can be expressed in terms of translational and rotational motion. The kinematic problem of robot depends on the linear velocity $\mathrm{v}$ and angular velocity $\omega$. The linear velocity of left and right wheel $\mathrm{v}_{\mathrm{L}}, \mathrm{v}_{\mathrm{R}}$ are calculated by angular velocity $\omega_{\mathrm{R}}$ and $\omega_{\mathrm{L}}$

$$
\begin{aligned}
& v_{R}=r * \omega_{R} \\
& v_{L}=r * \omega_{L}
\end{aligned}
$$

where $r$ : is radius of wheel.

The kinematics of the differential drive mobile robot is based on the assumption of pure rolling and there is no slip between the wheel and surface. The linear velocity $\mathrm{v}$ and angular velocity $\omega$ of robot is calculated as following equation

$$
\begin{gathered}
v=\frac{v_{x}+v_{y}}{2} \\
\omega=\frac{d \theta}{d t}=\frac{v_{R}-v_{L}}{d}
\end{gathered}
$$

where $\mathrm{d}$ : is distance between two wheels.

The tangential velocity according $\mathrm{x}$ axis and $\mathrm{y}$ axis is:

$$
\begin{aligned}
& \dot{x}=\frac{d x}{d t}=v \cos \theta \\
& \dot{y}=\frac{d y}{d t}=v_{R} \sin \theta
\end{aligned}
$$

The angular velocity $\omega$ is derivative of orientation of robot

$$
\dot{\theta}=\omega_{\mathrm{R}}
$$

In a nutshell, the kinematic model of robot is:

$$
\left[\begin{array}{c}
\dot{x} \\
\dot{y} \\
\dot{\theta}
\end{array}\right]=\left[\begin{array}{c}
v_{x} \\
v_{y} \\
\omega
\end{array}\right]=\left[\begin{array}{cc}
\cos \theta & 0 \\
\sin \theta & 0 \\
0 & 1
\end{array}\right]\left[\begin{array}{l}
v \\
\omega
\end{array}\right]
$$

where the tangential velocity in the $\mathrm{x}$ direction is $\dot{x}$ or $v_{x}$, the tangential velocity in the y direction is $\dot{y}$ or $v_{y}$, and the angular velocity is $\dot{\theta}$. By simple modification from (5), (6), (8), we get: 


$$
\left[\begin{array}{c}
\dot{x} \\
\dot{y} \\
\dot{\theta}
\end{array}\right]=\left[\begin{array}{c}
v_{x} \\
v_{y} \\
\omega
\end{array}\right]=\left[\begin{array}{cc}
\frac{1}{2} \cos \theta & \frac{1}{2} \cos \theta \\
\frac{1}{2} \sin \theta & \frac{1}{2} \sin \theta \\
0 & -1 / d
\end{array}\right]\left[\begin{array}{c}
\omega_{R} \\
\omega_{L}
\end{array}\right]
$$

This above kinematic equation is used for simulation in this research. This model is referred to as a kinematic model since it describes the velocities of the vehicle but not the forces or torques that cause the velocity.

\section{TRAJECTORY TRACKING OF MOBILE OF ROBOT USING MIMO FUZZY}

A behavior in a mobile robot navigation system usually represents a concern of the robot in which follow the path or avoid obstacles, target seeking are the most important task. Kinematics constraint in two dimensional workspaces is discussed in section II. Each robot has an image and an array of infrared sensors for measuring the distances of obstacles around it, and the bearing of the target and a radio system for communicating with other robots. The information's being sent among the robots are their positions, how far they are from the target, and whether reached the target or not. According to the information acquired by the robots using their sensors, some of the fuzzy control rules are activated accordingly.

The MIMO closed-loop control algorithm is demonstrated in this section for tracking trajectories. At each sample time, the calculation module calculate the error in location and orientation $\Delta \mathrm{x}, \Delta \mathrm{y}, \Delta \theta$ between the actual robot coordinates $(\mathrm{x}, \mathrm{y}, \theta)$ (which are received from sensors in the reality) and coordinates of the trajectory $\left(x_{d}, y_{d}, \theta_{d}\right)$. After that, the Distance and Angle is generated to be the inputs of Fuzzy controller. This controller is able to deliver appropriate action for mobile robot, velocity of left and right wheel $\left(\omega_{L}, \omega_{R}\right)$ as two outputs. To control the system, we assume the universe of discourse for the two state variables to be $0 \leq \mathrm{x}_{1}$ $($ Distance $) \leq 10(\mathrm{~cm}) ;-$ pi $\leq \mathrm{x}_{2}($ Angle $) \leq$ pi $(\mathrm{rad})$. The universe of discourse for the control velocity of left and right wheel is $0 \leq \mathrm{u}_{1}\left(\omega_{\mathrm{L}}\right) \leq 6 \mathrm{rad} / \mathrm{s}, 0 \leq \mathrm{u}_{2}\left(\omega_{\mathrm{L}}\right) \leq 6 \mathrm{rad} / \mathrm{s}$.

\section{A. Constructing the Membership Functions}

Five membership functions are considered with all are of triangular member. The languages are Zero $(Z)$, Near $(\mathrm{N})$, Medium (M), Far (F), Very Far (VF) and Big Negative (BN), Negative (N), Zero (Z), Positive (P), Positive Big (PB) for Distance, Angle respectively. Linguistic variables like are positive Very Slow (VS), Slow (S), Medium (M), Fast (F), Very Fast (VF) defined for $\omega_{L}, \omega_{R}$. The parameters defining the functions are listed in Table I. The membership functions describes above are depicted in Fig. 2

TABLE I. PARAMETERS FOR INPUTS AND OUTPUTS FUZZY CONTROLLER

\begin{tabular}{|c|c|c|c|c|c|}
\hline Variables & $Z$ & $\mathrm{~N}$ & M & $\mathrm{F}$ & VF \\
\hline $\mathrm{X}_{1}(\mathrm{~cm})$ & {$\left[\begin{array}{lll}0 & 0 & 2.5\end{array}\right]$} & {$\left[\begin{array}{lll}0 & 2.5 & 5\end{array}\right]$} & $\begin{array}{l}{\left[\begin{array}{l}2.55 \\
7.5]\end{array}\right.} \\
\text { ? }\end{array}$ & $\begin{array}{l}{\left[\begin{array}{ll}5 & 7.5 \\
10]\end{array}\right.} \\
\end{array}$ & $\begin{array}{l}{\left[\begin{array}{ll}7.5 & 10 \\
12.5\end{array}\right]} \\
\end{array}$ \\
\hline $\mathrm{X}_{2}(\mathrm{rad})$ & $\begin{array}{l}\mathrm{BN} \\
{\left[\begin{array}{lll}-1 & -0.5 & -0.1\end{array}\right]}\end{array}$ & $\begin{array}{lll}\mathrm{N} \\
{[-0.5} & -0.1 \\
0] & \\
\end{array}$ & \begin{tabular}{|l}
$Z$ \\
{$\left[\begin{array}{ll}-0.1 & 0 \\
0.1\end{array}\right]$} \\
\end{tabular} & $\begin{array}{ll}\mathrm{P} \\
{\left[\begin{array}{ll}0 & 0.1 \\
0.5\end{array}\right]} \\
\end{array}$ & $\begin{array}{l}\mathrm{BP} \\
{\left[\begin{array}{llll}0.1 & 0.5 & 1\end{array}\right]}\end{array}$ \\
\hline $\mathrm{U}_{1}, \mathrm{U}_{2}(\mathrm{rad} / \mathrm{s})$ & $\begin{array}{l}\mathrm{VS} \\
{\left[\begin{array}{lll}0 & 0 & 1.5\end{array}\right]}\end{array}$ & $\begin{array}{l}\mathrm{S} \\
{\left[\begin{array}{lll}0 & 1.5 & 3\end{array}\right]}\end{array}$ & $\begin{array}{l}M \\
{\left[\begin{array}{ll}1.5 & 3 \\
4.5]\end{array}\right.}\end{array}$ & $\begin{array}{l}\mathrm{F} \\
{[3}\end{array}$ & $\begin{array}{l}\text { VF } \\
{\left[\begin{array}{lll}4.5 & 6 & 7.5\end{array}\right]}\end{array}$ \\
\hline
\end{tabular}

\section{B. Constructing the Rule Base}

In order to satisfy the control objective it is necessary to design a fuzzy logic control for the real velocities of the mobile robot which use linguistic variables in the inputs and outputs. After the stage of constructing membership functions, we construct the FAM (Fuzzy Associative Memory) table for the rule bases, which is shown in Table II. The expert system is developed based on the IF- THEN rules as 25 following rules to control robot tracking defined trajectory. For example the first rule is:

IF (Angle is Big Negative and Distances is Zero) THEN (Velocity of right wheel is Very slow and Velocity of left wheel is Very Fast)

This rule will help robot change the direction in the meanwhile try to keep the tracking desired position. The inference output from these rules is computed by Mamdani (max-min) operator for composition, minimum operation for implication, and center of area for defuzzification as shown in Fig. 3.

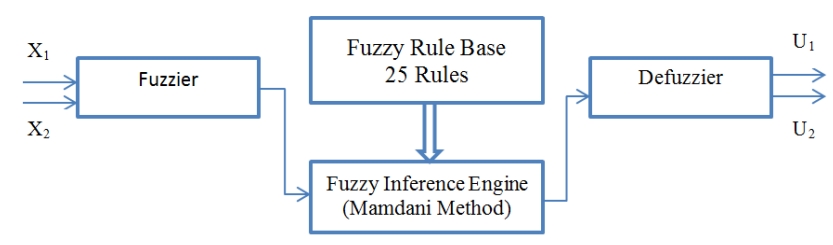

Figure 2. Methods of the conventional tracking fuzzy control system

TABLE II. RULES OF RIGHT WHEEL VELOCITY U1

\begin{tabular}{cccccc}
\hline & \multicolumn{5}{c}{$\mathbf{X}_{\mathbf{1}}$} \\
$\mathbf{X}_{\mathbf{2}}$ & $\mathbf{B N}$ & $\mathbf{N}$ & $\mathbf{Z}$ & $\mathbf{P}$ & $\mathbf{B P}$ \\
\hline $\mathbf{Z}$ & VS & S & VS & VF & VF \\
$\mathbf{N}$ & VS & VS & S & VF & VF \\
$\mathbf{M}$ & VS & S & M & VF & VF \\
$\mathbf{F}$ & VS & S & F & VF & VF \\
VF & VS & S & VF & VF & VF \\
\hline
\end{tabular}

TABLE III. RULES OF LEFT WHEEL VELOCITY U2

\begin{tabular}{llllll}
\hline & \multicolumn{5}{c}{$\mathbf{X}_{\mathbf{1}}$} \\
\cline { 2 - 5 } $\mathbf{X} 2$ & $\mathbf{B N}$ & $\mathbf{N}$ & $\mathbf{Z}$ & $\mathbf{P}$ & $\mathbf{B P}$ \\
\hline $\mathbf{Z}$ & VF & VF & VS & S & VS \\
$\mathbf{N}$ & VF & VF & S & S & VS \\
$\mathbf{M}$ & VF & VF & M & S & VS \\
$\mathbf{F}$ & VF & VF & F & S & VS \\
VF & VF & VF & VF & S & VS \\
\hline
\end{tabular}

\section{Simulation Results and Discussion}

MATLAB Fuzzy Logic Toolbox is used to design of fuzzy logic controller (FLC). The toolbox contains functions, graphical user interfaces and data structures that allow the user to quickly design, test, simulate and modify a fuzzy inference system. 
The steps in FLC design are described in this section. To validate the implemented tracking trajectory MIMO fuzzy controller, seven design paths is tested include: zig - zag line, rectangular, Trapezium, Circle, Elip and eight shapes. Initial position of robot is $(0,0)$ for all situation. The program is written in Matlab as Fig. 4, the designed trajectory is given in the left hand side block. Those values are compared with actual feedback value, then we use Matlab function to calculate the value of $\mathrm{x}_{1}$ (distance variable) and $x_{2}$ (angle variable). The fuzzy controller includes two inputs are $\mathrm{x}_{1}$ and $\mathrm{x}_{2}$. The two outputs are velocity of right wheel $\left(\mathrm{u}_{1}\right)$ and velocity of left wheel $\left(\mathrm{u}_{2}\right)$ The Mobile robot is written for kinematic of the robot using equation (11) in section II. After this block, the values of orientation and position of robot are computed and used as feedback signals for whole system. Fig. 5 depicts the simulation results for tracking trajectories which use MIMO fuzzy controller. The blue path is design trajectory and the red path is robot's tracking trajectory.

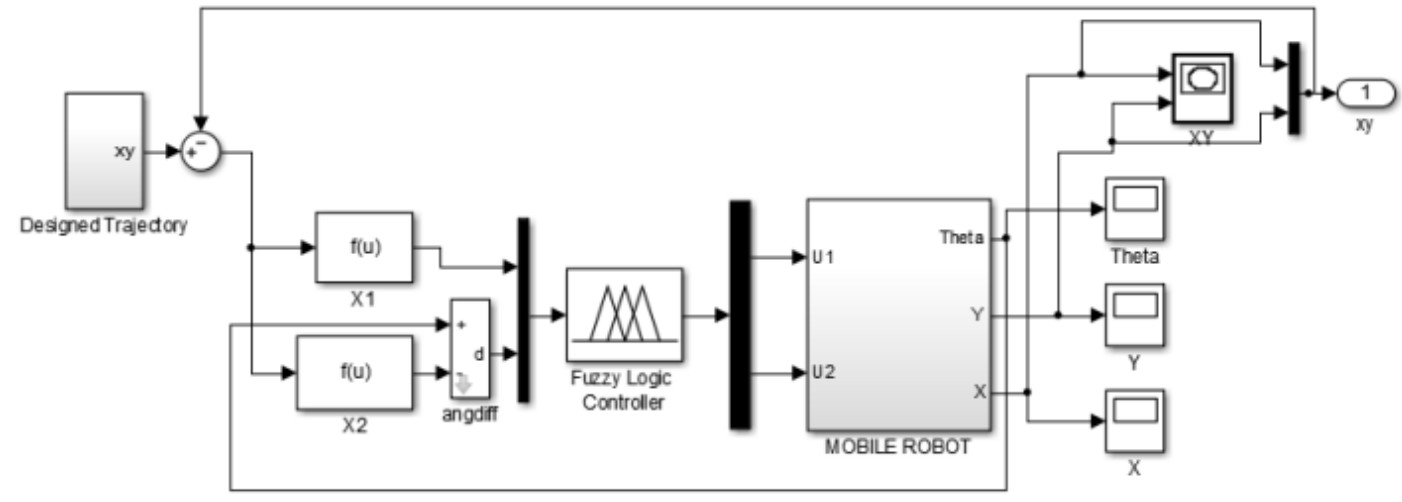

Figure 3. MIMO Fuzzy controller for mobile robot tracking trajectory

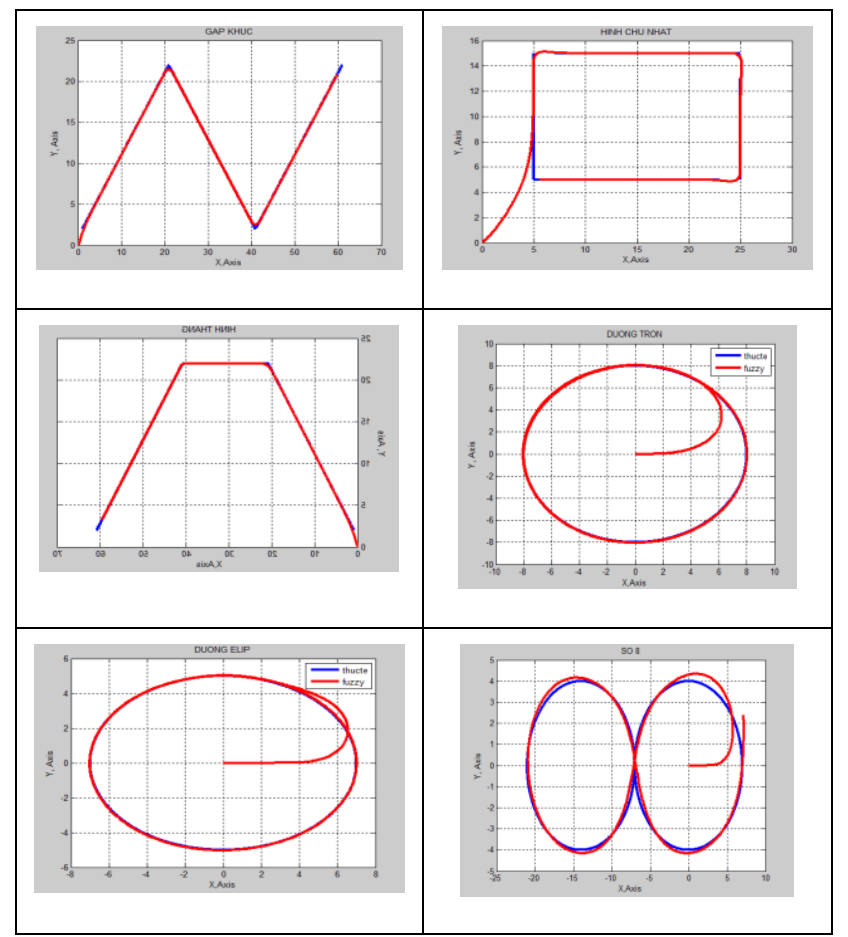

Figure 4. Tracking trajectory simulation results

From the simulation results, MIMO fuzzy controller are successful to apply for tracking behavior of mobile robot with the error is acceptable.

\section{APPLY MIMO FUZZY CONTROLLER FOR ROBOT NAVIGATION}

In this section, a new intelligent controller has been proposed for mobile robot navigation using MIMO fuzzy logic. It is more efficient than the other traditional reactive behavior control and also easier to design and implement. In our MIMO controller, the target seeking behavior and obstacle avoidance is combining together.

\section{A. Target Seeking Behavior}

First step, the robot can adjusts its motion direction and moves towards the target since it know current position and find the target position. The distance between robot and its destination is

$$
\mathrm{d}=\sqrt{\left(y_{\text {Target }}-y_{\text {Robot }}\right)^{2}+\left(x_{\text {Target }}-x_{\text {Robot }}\right)^{2}}
$$

The robot also need adjust its orientation when it reaches the goal point. Assume that the angle between robot and $\mathrm{x}$ axis is theta $(\theta)$; the angle between robot and target is beta $(\beta)$ in $\mathrm{x}$ direction so that the angle must be changed is gama $(\gamma)$

$$
\beta=\operatorname{atan} \frac{y_{\text {Target }}-y_{\text {Robot }}}{x_{\text {Target }}-x_{\text {Robot }}} ; \gamma=\beta-\theta
$$

\section{B. Obstacle Avoidance}

In case, the workspace of robot is free, robot moves fast and reduce speed when the end point nearby; it will stop there. When the acquired information from the sensors shows that there exist obstacles nearby robot, it must decrease its speed to avoid obstacles. When a robot is close to an obstacle, it must it must change its speed and steering angle to avoid the obstacle. When the robot sense obstacle near to it or the robot moves at curved and narrow roads, it must reduce its speed to avoid collision with obstacles. The fuzzy rules are obtained heuristically In this case; its main reactive behavior is decelerating for obstacle avoidance. 


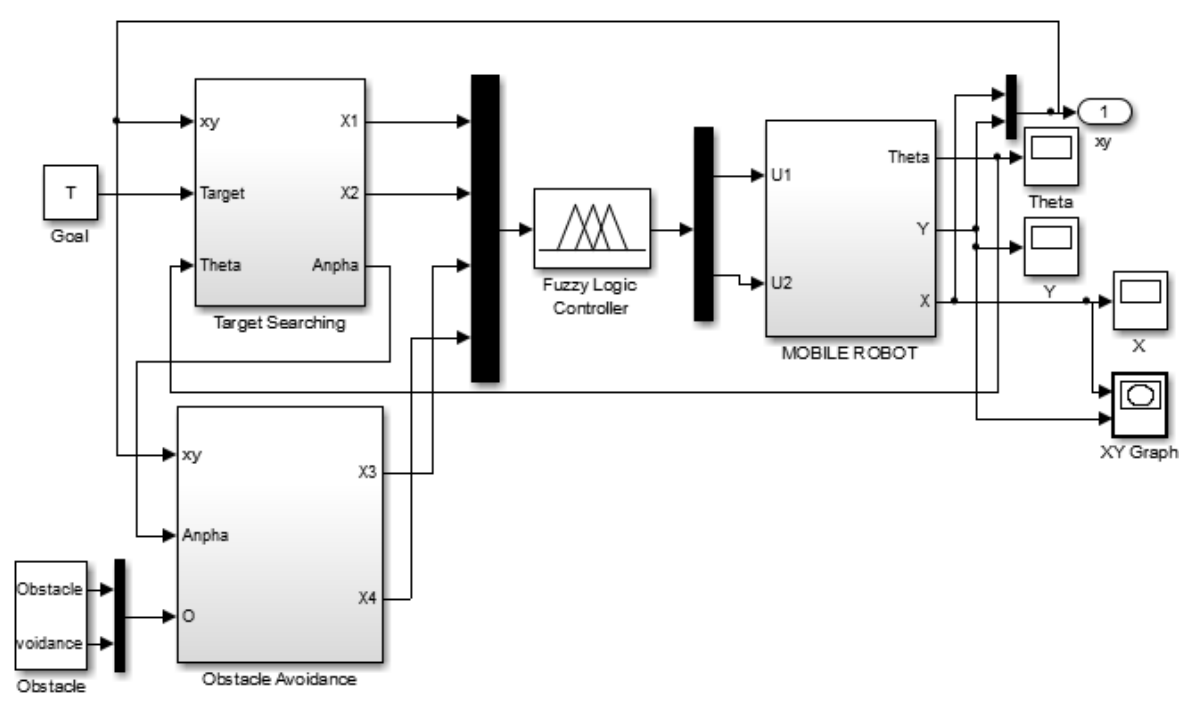

Figure 5. MIMO fuzzy controller for mobile robot navigation

The Mobile Robot Navigation fuzzy control system is based on the MIMO closed-loop fuzzy system shown in Fig. 5. Similar section III, to control the system, we assume the universe of discourse for the four state variables to be $0 \leq \mathrm{x}_{1}$ (Distance between robot and target) $\leq 10(\mathrm{~cm}) ;-$ pi $\leq \mathrm{x}_{2}$ (Angle between robot and target $) \leq$ pi $(\mathrm{rad}) ; 0 \leq \mathrm{x}_{3}$ (Distance between robot and Obstacles) $\leq 10$ (cm); - $\mathrm{pi} \leq \mathrm{x}_{4}$ (Angle between robot and Obstacles) $\leq$ pi (rad). The universe of discourse for the control velocity of left and right wheel is $0 \leq \mathrm{u}_{1}\left(\omega_{\text {right }}\right) \leq 6 \mathrm{rad} / \mathrm{s}, 0 \leq \mathrm{u}_{2}$ $\left(\omega_{\text {left }}\right) \leq 6 \mathrm{rad} / \mathrm{s}$.

The Goal and Obstacle block includes the position of Target and Obstacles in $\mathrm{x}$ and $\mathrm{y}$ dimension. Base on those values the target searching and Obstacle Avoidance block will calculate the inputs for fuzzy controller.

The MIMO Fuzzy controller for navigation of mobile robot is implemented for three membership functions for each input as illustrate in Fig. 6. The linguistic variables for fuzzy rules are Near (N), Medium (M), Far (F) for $x_{1}$, $\mathrm{x}_{3}$ and Negative $(\mathrm{N})$, Zero $(\mathrm{Z})$, Positive $(\mathrm{P})$ for $\mathrm{x}_{2}, \mathrm{x}_{4}$ which are the velocity of left and right wheel of mobile robot.

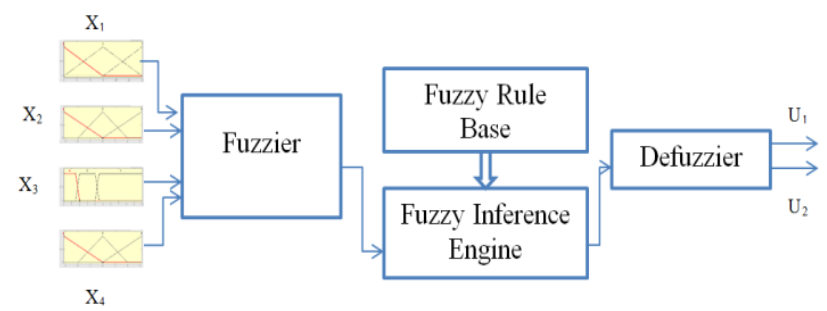

Figure 6. Methods of the conventional navigation fuzzy control system

Fig. 8 and Fig. 9 illustrate the path performed path td by the mobile robot to reach the target. The generated way to the destination is relatively smooth. The robot speed varies with respect to its distance, angle between robot and target as well as with obstacles by fuzzy logic controller.

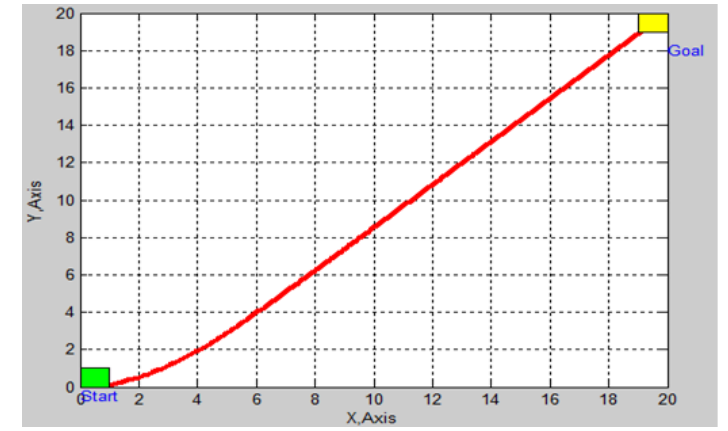

Figure 7. Reaching target without obstacles

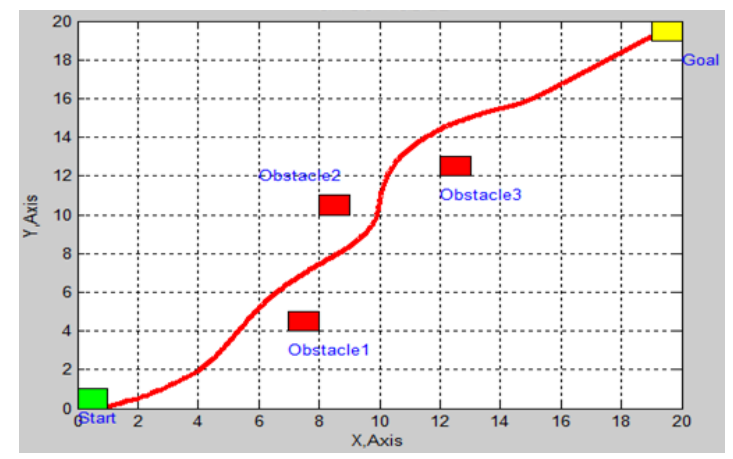

Figure 8. Reaching target with obstacles

In all scenarios, the robot moves from the initial point to the end point with suitable velocity. In Fig. 8, three obstacles have been added to the scene. As we can see, the motion of robot in the workspace with and without obstacles is different. The adjustment in path planning of robot because of avoiding the obstacles in second scenario is applied by MIMO fuzzy controller.

\section{CONCLUSION}

We have presented in this work the application of MIMO fuzzy path-following and navigation control for mobile robot. The strategy presents a desired performance as it is able to accurately follow the different 
designed trajectory which makes it desired for the case of working in indoor or conned environments as well as decide the path planning to avoid obstacles and go to target. The simulation results are provided to illustrate the feasibility and effectively of the proposed MIMO fuzzy controller.

\section{REFERENCES}

[1] G. Antonelli, S. Chiaverini, and G. Fusco, "A fuzzy-logic-based approach for mobile robot path tracking," IEEE Transactions on Fuzzy Systems, vol. 15, ch. 2, pp. 211-221, 2007.

[2] T. H. Lee, H. K. Lam, F. H. F. Leung, and P. K. S. Tam, "A practical fuzzy logic controller for the path tracking of wheeled mobile robots," IEEE Control Systems Magazine, pp. 60-65, 2003.

[3] C. F. Juang and C. H. Hsu, "Reinforcement ant optimized fuzzy controller for mobile robot wall following control," IEEE Transactions on Industrial Electronics, pp. 56, 2009.

[4] D. R. Parhi, "Navigation of mobile robots using a fuzzy logic controller," Journal of Intelligent and Robotic Systems, vol. 42, pp. 253-273, 2005.

[5] W. S. Lin, C. L. Huang, and M. K. Chuang, "Hierarchical fuzzy control for autonomous navigation of wheeled robots," IEEE Proc Control Theory Application, vol. 152, ch. 5, pp. 598-606, 2005.

[6] D. R. Parhi and M. K. Singh, "Intelligent fuzzy interface technique for controller of mobile robot," Journal of Mechanical Engineering Science, part C, vol. 222, ch. 11, pp. 2281-2292, 2008

[7] M. Faisal, R. Hedjar, M. A. Sulaiman, and K. Al-Mutib, "Fuzzy logic navigation and obstacle avoidance by a mobile robot in an unknown dynamic environment," International Journal of Advanced Robotic Systems, vol. 10, 2013.

[8] K. P. Mohanty and R. P. Dayal "Navigation of autonomous mobile robot using adaptive network based fuzzy inference system," Journal of Mechanical Science and Technology, vol. 28, no. 7, pp. 2861-2868, July 2014.

[9] T. Das and I. N. Kar, "Design and implementation of an adaptive fuzzy logic-based controller for wheeled mobile robots," IEEE Transactions on Control Systems Technology, vol. 14, ch. 3, pp. 501-510, 2006.

[10] S. Tong, B. Chen, and Y. Wang, "Fuzzy adaptive output feedback control for MIMO nonlinear systems," Fuzzy Sets and Systems, vol. 156 , no. 2 , pp. 285-299, 2005

[11] H. X. Li and S. Tong, "A hybrid adaptive control for a class of nonlinear MIMO systems," IEEE Transactions on Fuzzy Systems, vol. 11, no. 1, pp. 24-34, 2003.

[12] E. Kim and L. Sungryul, "Output feedback tracking control of MIMO systems using a fuzzy disturbance observer and its applications to the speed control of a PM synchronous motor," IEEE Transactions on Fuzzy Systems, vol. 13, no. 6, pp. 725-741, 2005.

[13] Q. Feng, Q. Zhu, A. F. T. Winfield, and C. Melhuish, "Adaptive sliding mode control for MIMO nonlinearsystems based on fuzzy logic scheme," International Journal of Automation and Computing, vol. 1, pp. 51-62, 2004.

[14] N. Paykari, S. H. Abbasi, and F. Shabaninia "Design of MIMO mamdani fuzzy logic controllers for wall following Mobile robot," Advances in Intelligent Systems and Computing, vol. 195, pp. 155164, 2013.

Thoa T. Mac received the B.Eng. degree in mechatronics engineering from Hanoi University of Science and Technology, Vietnam, in 2006. She obtained her master degree in 2009 at National Taiwan university of Science and Technology in Faculty of Engineering, Taiwan. Now she is a research $\mathrm{PhD}$ student both in Hanoi University of Science and Technology, Vietnam and Ghent University in Mechatronics. Her interests include fuzzy logic systems, neural networks, trajectory planning and intelligent control for autonomous robot, optimization.

Cosmin Copot received his M.Sc. and M.E. degrees in systems engineering from Gheorghe Asachi Technical University of Iasi, Romania, in 2007, and 2008, respectively. In 2011 he received Ph.D. degree from the same university on control techniques for visual servoing systems. He is currently a post-doctoral research at Ghent University. His research interests include robotics, visual servoing systems, identification and control.

Prof. Dr. Ir. Robin De Keyser received the M.Sc. degree in electromechanical engineering and the Ph.D. degree in control engineering from Ghent University, Ghent, Belgium, in 1974 and 1980, respectively. $\mathrm{He}$ is currently a Full Professor of control engineering with the Faculty of Engineering, Ghent University. He is the author or co-author of over 300 publications in journals, books, and conference proceedings. He has acted as an external review expert in several European Commission Research Programs and is one of the pioneers who produced the original concepts of predictive control during the 1980s. The research is application-driven, with many pilot implementations in technical and nontechnical systems, amongst others in the chemical, steel, marine, mechatronic, semiconductor, power electronics, and biomedical spheres. His current research interests include model predictive control, autotuning and adaptive control, modeling and simulation, and system identification. 